\title{
Revenge of the killer microbe
}

\author{
Sandra R. Arnold MD MSc
}

$\infty$

See related article page 877

$\mathrm{T}$ he cover of Time Magazine on Sept. I4, 1994, read "Revenge of the killer microbe" in reference to an article about emerging infections, including drug-resistant bacteria that are difficult to treat. ${ }^{1} \mathrm{~A}$ lead article in Science just 2 years earlier cautioned readers about the possibility of a "post-antibiotic era" and stressed the importance of the discovery of new drugs and the prudent use of currently available antimicrobials. ${ }^{2}$ The mantra of the ills of antibiotic overuse has been repeated in the medical and lay press over the past IO-I5 years.

It is clear from ecologic studies that antibiotic use is a driving force in the emergence of antibiotic-resistant organisms in community and hospital settings. Both the inappropriate use of antibiotics for viral respiratory tract infections and the overuse of powerful, broad-spectrum antibiotics contribute substantially to this problem. Despite the long-term trend of decreasing rates of antibiotic use for common infections in outpatient settings, it remains imperative that we continue to reduce inappropriate antibiotic use if there is any possibility of slowing the further development of drug resistance in common pathogens.

Physicians who are surveyed or interviewed uniformly identify patient and parent expectations for antibiotic therapy as being the strongest influence on unnecessary prescribing of antibiotics. ${ }^{3,4}$ Indeed, physicians are more likely to prescribe antibiotics if they believe that the patient or parent expects a prescription for antibiotics. ${ }^{5}$ The accuracy of physicians' interpretation of patients' demands is poor. ${ }^{6}$ Surveys and interviews with patients and parents reveal that, although they frequently expect antibiotics to be prescribed, they expect physicians to explain the illness and want to feel that their physician has heard and addressed their concerns. ${ }^{6}$

Studies have demonstrated that a variety of physician factors are associated with inappropriate antibiotic prescribing, including involvement in medical education, time since graduation or time in practice, specialty, location of training, prescribing rates for other drugs, location of practice (urban $v$. rural) and practice volume. ${ }^{7-9}$ Physician knowledge and understanding of the diagnosis, as well as of the management of community-acquired infections, has also been suggested to influence the decision to prescribe and the selection of antibiotics. These factors certainly contribute to the large variation in practice among physicians in terms of the decision to prescribe and the selection of antibiotics.

Changing physician practice has proven extremely difficult for many behaviours, including prescribing..$^{10}$ If we want to effect further reductions in inappropriate antibiotic prescribing, greater understanding of the physician decision-making

\section{Key points of the article}

- Rates of inappropriate antibiotic prescribing (prescription of antibiotics for viral infections or of second- or third-line antibiotics as first-line treatment) have decreased since the early to mid-1990s; however, physicians continue to prescribe antibiotics inappropriately at unacceptable rates

- Cadieux and colleagues, using a retrospective cohort design, have demonstrated that international medical graduates, physicians with high-volume practices and those in practice longer are more likely to prescribe antibiotics inappropriately

- They were not able to link inappropriate antibiotic prescribing to knowledge of either general medicine or infectious diseases (measured by scores on the Medical Council of Canada Qualifying Examination)

- Physician uncertainty about the cause (bacterial v. viral) of infection may drive "just in case" prescribing

- Targeted interventions to improve antibiotic prescribing should address physician uncertainty. Interventions may include the use of near-patient diagnostic tests and personal practice audits for maintenance of certification

process and of the factors that influence antibiotic prescribing is needed.

In this issue of $C M A J$, Cadieux and colleagues ${ }^{11}$ examine $^{2}$ the association of physician knowledge (estimated by the use of scores obtained on the Medical Council of Canada Qualifying Examination [overall score and infectious diseases subscore]), time in practice (time since graduation from medical school), place of training and practice volume with the decision to prescribe and the choice of antibiotics. Using 4 health databases in Quebec, the authors evaluated the prescribing practices of a cohort of physicians who obtained their licences between 1990 and 1993 . Their findings suggest that the longer a physician is in practice, the greater the propensity is to prescribe antibiotics inappropriately. ${ }^{11}$ They found that the number of prescriptions for viral infections increased by $4 \%$ each year after graduation and that inappropriate prescription of second- and third-line antibiotics increased by $\mathrm{I} \%$ per year. In addition, Cadieux and colleagues found that international medical graduates and physicians who see more patients per day prescribe more antibiotics inappropriately. ${ }^{11}$ However, they did not find an association between physician knowledge

Sandra Arnold is an associate professor in the Department of Pediatrics, Division of Infectious Diseases, University of Tennessee Health Science Center, Le Bonheur Children's Medical Center, Memphis, Tenn. 
of infectious diseases and inappropriate antibiotic prescribing. This probably relates to the measure chosen to estimate physician knowledge, which was the score obtained on the Medical Council of Canada Qualifying Examination. Despite the previous findings by Tamblyn and colleagues that scores on qualifying examinations are associated with quality of care in practice, this does not hold true for the use of antibiotics. ${ }^{12}$

The factors that figure into the decision to prescribe and the choice of antibiotic are not simply related to physician knowledge of the underlying disease pathogenesis or its natural history. True, there are knowledge gaps related to the clinical presentation and progression of viral infections, such as the diagnostic utility of purulent discharges; ${ }^{13}$ however, it has been shown that physicians do not approach antibiotic prescribing decisions in the same way that they approach other management decisions in practice, including prescribing other medications. Physicians' actions often prove to be contrary to their stated intentions and what they believe, in general, to be the correct decision. ${ }^{14}$ Why is it, then, that physicians continue to prescribe antibiotics for viral infections knowing that antibiotics will not hasten recovery and knowing that there are important patient and societal ill effects that may result from the inappropriate use of antibiotics?

Qualitative research suggests that, for many physicians, the decision to prescribe antibiotics to individual patients relates to the perceived risk for complications and the potential, however slight, for an immediate benefit, and that the perceived risks and benefits outweigh future risks to the individual or to society. ${ }^{3,4}$ In short, physicians may be unwilling to tolerate the low risk of missing a bacterial diagnosis and choose to err on the side of caution and prescribe antibiotics. In the current study by Cadieux and colleagues, only $22 \%$ of patients with laryngitis received a prescription, compared with $43 \%$ of those with influenza. Patients with influenza tend to appear quite unwell, and some patients, especially those who are elderly, are at risk of complicating bacterial pneumonia. Thus, despite the known viral etiology of this illness, the uncertainty about a patient's condition at the time of diagnosis, and over the ensuing days, may prompt a prescription.

Where does this leave us? Unfortunately, the simple provision of education to physicians about common infections for which antibiotics are inappropriate does not target this problem. We know from systematic reviews of interventions to change physician behaviours ${ }^{10}$ that there are no "magic bullets." A Cochrane Collaboration systematic review of interventions to improve antibiotic prescribing in ambulatory settings found that no intervention, or combination of interventions, consistently produced large reductions in antibiotic use. ${ }^{15}$ In this review, interventions that targeted identified barriers to changes in behaviour were generally more successful than other interventions. ${ }^{15}$ However, devising educational interventions that address physician uncertainty with a diagnosis will be considerably more difficult than simply re- minding physicians that viral infections do not improve with antibiotic therapy.

Effective interventions might include education about the appropriate use of point-of-care diagnostic testing, including rapid streptococcal and influenza testing in the office setting, to reduce diagnostic uncertainty. The use of rapid tests to diagnose influenza in emergency-department settings has been shown to reduce the number of antibiotic prescriptions dispensed. ${ }^{16}$ The creation of an antibiotic stewardship module for primary care specialties to fulfill the requirement for practice audit in maintenance of certification programs would allow physicians to examine the reasons for their own practice decisions and to observe outcomes in treated and untreated patients. We must also continue to beat the drum with the old educational messages in order to sustain the improvements in prescribing that have already been attained and to provide ongoing education to new and old graduates and to the general public.

Competing interests: None declared.

\section{REFERENCES}

I. Lemonick MD. The killers all around. Time I994;I44:62

2. Cohen ML. Epidemiology of drug resistance: implications for a post-antibiotic era. Science I992;257:1050-5.

3. Butler CC, Rollnich S, Pill R, et al. Understanding the culture of prescribing: qualitative study of general practitioners' and patients' perceptions of antibiotic for sore throats. BMJ I998;317:637-42.

4. Kumar S, Little P, Britten N. Why do general practitioners prescribe antibiotics for sore throat? Grounded theory interview study. BMJ 2003;326:138.

5. Mangione-Smith R, McGlynn EA, Elliott MN, et al. Parent expectations for antibiotics, physician-parent communication, and satisfaction. Arch Pediatr Adolesc Med 200I; $155: 800-6$.

6. Hamm RM, Hicks RJ, Bemben DA. Antibiotics and respiratory infections: Are patients more satisfied when expectations are met? J Fam Pract I996;43:56-62.

7. Arnold SR, To T, McIsaac WJ, et al. Antibiotic prescribing for upper respiratory tract infection: the importance of diagnostic uncertainty. J Pediatr 2005;146:222-6.

8. Hutchinson JM, Foley RN. Method of physician remuneration and rates of antibiotic prescription. CMAJ I999; I60:I0I3-7.

9. Nash DR, Harman J, Wald ER, et al. Antibiotic prescribing by primary care physicians for children with upper respiratory tract infections. Arch Pediatr Adolesc Med 2002; I56:III4-9.

Io. Oxman $\mathrm{AD}$, Thomson MA, Davis DA, et al. No magic bullets: a systematic review of IO2 trials of interventions to improve professional practice. $C M A J$ I995;I53:I423-3I.

II. Cadieux G, Tamblyn R, Dauphinee D, et al. Predictors of inappropriate antibiotic prescribing among primary care physicians. CMAJ 2007;177:877-83.

I2. Tamblyn R, Abrahamowicz M, Brailovsky C, et al. Association between licencing examination scores and resource use and quality of care in primary care practice. JAMA ז998;280:989-96.

I3. Gonzales R, Barrett PH, Steiner JF. The relation between purulent manifestations and antibiotic treatment of upper respiratory tract infections. J Gen Intern Med I999;I4:I5I-6.

I4. Lambert BL, Salmon JW, Stubbings J, et al. Valuck rJ, Kezlarian K. Factors associated with antibiotic prescribing in a managed care setting: an exploratory investigation. Soc Sci Med 1997;45:1767-9.

I5. Arnold SR, Straus SE. Interventions to improve antibiotic prescribing practices in ambulatory care [review]. Cochrane Database Syst Rev 2005:CDo03539.

I6. Sharma V, Dowd D, Slaugher AJ, et al. Effect of rapid diagnosis of influenza virus type A on the emergency department management of febrile infants and toddlers. Arch Pediatr Adolesc Med 2002;156:4I-3.

Correspondence to: Dr. Sandra R. Arnold, Division of Infectious

Diseases, University of Tennessee Health Science Center, Le

Bonheur Children's Medical Center, 50 Dunlap St. N, Memphis

TN38103; sarnold@utmem.edu 